NBER WORKING PAPER SERIES

\title{
INTERNET ADVERTISING AND THE GENERALIZED SECOND PRICE AUCTION: SELLING BILLIONS OF DOLLARS WORTH OF KEYWORDS
}

\author{
Benjamin Edelman \\ Michael Ostrovsky \\ Michael Schwarz \\ Working Paper 11765 \\ http://www.nber.org/papers/w11765
}

\author{
NATIONAL BUREAU OF ECONOMIC RESEARCH \\ 1050 Massachusetts Avenue \\ Cambridge, MA 02138 \\ November 2005
}

\footnotetext{
We are grateful to David Pennock and Yahoo! for data and advice. We also thank Drew Fudenberg, Louis Kaplow, David McAdams, Paul Milgrom, Muriel Niederle, Ariel Pakes, and Al Roth for helpful discussions. The views expressed herein are those of the author(s) and do not necessarily reflect the views of the National Bureau of Economic Research.

(C)2005 by Benjamin Edelman, Michael Ostovsky, and Michael Schwarz. All rights reserved. Short sections of text, not to exceed two paragraphs, may be quoted without explicit permission provided that full credit, including () notice, is given to the source.
} 
Internet Advertising and the Generalized Second Price Auction: Selling Billions of Dollars Worth of Keywords

Benjamin Edelman, Michael Ostovsky, and Michael Schwarz

NBER Working Paper No. 11765

November 2005

JEL No. L0

\title{
ABSTRACT
}

We investigate the "generalized second price" auction (GSP), a new mechanism which is used by search engines to sell online advertising that most Internet users encounter daily. GSP is tailored to its unique environment, and neither the mechanism nor the environment have previously been studied in the mechanism design literature. Although GSP looks similar to the Vickrey-ClarkeGroves (VCG) mechanism, its properties are very different. In particular, unlike the VCG mechanism, GSP generally does not have an equilibrium in dominant strategies, and truth-telling is not an equilibrium of GSP. To analyze the properties of GSP in a dynamic environment, we describe the generalized English auction that corresponds to the GSP and show that it has a unique equilibrium. This is an ex post equilibrium that results in the same payoffs to all players as the dominant strategy equilibrium of VCG.

\author{
Benjamin Edelman \\ Department of Economics \\ Harvard University \\ Cambridge, MA 02138 \\ bedelman@fas.harvard.edu \\ Michael Ostrovsky \\ Graduate School of Business \\ Stanford University \\ Stanford, CA 94305 \\ ostrovsky@gsb.stanford.edu \\ Michael Schwarz \\ University of California, Berkeley \\ 140 Warren Hall MC 7360 \\ Berkeley, CA 947210-7360 \\ and NBER \\ mschwarz@berkeley.edu
}




\section{Introduction}

This paper investigates a new auction mechanism, which we call the "generalized second price" auction, or GSP. GSP is tailored to the unique environment of the market for online ads, and neither the environment nor the mechanism have previously been studied in the mechanism design literature. While studying the properties of a novel mechanism is often fascinating in itself, our interest is also motivated by the spectacular commercial success of GSP. It is the dominant transaction mechanism in a large and rapidly growing industry. For example, Google's total revenue in 2004 was equal to $\$ 3.189$ billion. Over $98 \%$ of the revenue came from GSP auctions. Yahoo!'s total revenue in 2004 was equal to $\$ 3.574$ billion. It is believed that over 50\% of Yahoo!'s revenue is derived from sales via GSP auctions. To appreciate the size of the market dominated by GSP auctions, note that the combined market capitalization of Google and Yahoo! is over $\$ 125$ billion. In comparison, the combined market capitalization of all US airlines is about $\$ 20$ billion.

Let us briefly describe how these auctions work. When an Internet user enters a search ("query") into a search engine, he gets back a page with results, containing both the links most relevant to the query and the sponsored links, i.e., paid advertisements. The ads are clearly distinguishable from the actual search results, and different searches yield different sponsored links: advertisers target their ads based on search keywords. For instance, if a travel agent buys the word "Hawaii," then each time a user performs a search on this word, a link to the travel agent will appear on the search results page. When a user clicks on the sponsored link, he is sent to the advertiser's web page. The advertiser then pays the search engine for sending the user to its web page, hence the name - "pay-per-click" pricing.

The number of ads that the search engine can show to a user is limited, and different positions on the search results page have different desirabilities for advertisers: an ad shown at the top of a page is more likely to be clicked than an ad shown at the bottom. Hence, search engines need a system for allocating the positions to advertisers, and auctions are a natural choice. Currently, the mechanisms most widely used by search engines are based on GSP.

In the simplest GSP auction, for a specific keyword, advertisers submit bids stating their maximum willingness to pay for a click. When a user enters a keyword, he receives search results along with sponsored links, the latter shown in decreasing order of bids. In particular, the ad with the highest bid is displayed at the top, the ad with the next highest bid is displayed in the second position, and so on. If a user subsequently clicks on an ad in position $k$, that advertiser is charged by the search engine an amount equal to the next highest bid, i.e., the bid of an advertiser in position $k+1$. If a search engine offered only one advertisement per result page, this mechanism would be equivalent to the standard second price, or Vickrey-Clarke-Groves (VCG), auction. With multiple positions available, the GSP generalizes the second price auction. (Hence the name.) Here, a winner pays the next highest bidder's bid. But as we will demonstrate, the multi-unit GSP auction is no longer equivalent to the VCG auction, for multi-unit GSP lacks some of VCG's desirable properties. In particular, unlike the VCG mechanism, GSP generally does not have an equilibrium in dominant strategies, and truth-telling is not an equilibrium of GSP. 
In Section 2, we describe the evolution of the market for Internet advertisements and the unique features of the environment in this market. In Section 3, we introduce a model of sponsored search auctions, and we begin our analysis of the model in Section 4. Since advertisers can change their bids frequently, sponsored search auctions can be modeled as a continuous or an infinitely repeated game. Our analysis begins with a static stage game. We introduce restrictions on bidders' behavior suggested by the market's dynamic structure. We call the static equilibria satisfying these restrictions "locally envy-free."

We then proceed to show that the set of locally envy-free equilibria contains an equilibrium in which the payoffs of the players are the same as in the dominant-strategy equilibrium of the VCG auction, even though both the bids of the players and the payment rules in the mechanisms are very different. Moreover, this equilibrium is the worst locally envy-free equilibrium for the search engine and the best locally envy-free equilibrium for the bidders. Consequently, in any locally

envy-free equilibrium of GSP, the total expected revenue to the seller is at least as high as in the dominant-strategy equilibrium of the VCG auction.

In Section 5, we present our main result. The generalized English auction can be viewed as a dynamic game corresponding to the GSP auction. Although the generalized English auction is not dominant-strategy solvable, it has a unique perfect Bayesian equilibrium in continuous strategies. In this equilibrium, all players receive the same payoffs as in the dominant strategy equilibrium of VCG. A remarkable feature of the equilibrium of the generalized English auction is that it is an ex post equilibrium, i.e., the outcome depends only on the values of the participants and not on their beliefs about each others' values. Section 6 concludes.

\section{The Structure and Evolution of Sponsored Search Auctions}

\subsection{Unique Features of the Market for Internet Advertising}

A combination of several features makes the market for Internet advertising unique. First, bidding takes place continuously. For example, the advertiser with the second highest bid on a given keyword at some instant will be listed as the second sponsored link at that instant. But any other advertiser can revise his bid at any time, and the order of sponsored links and prices will change accordingly. These changes can be very rapid because advertisers can employ automated robots, including commercially available software, in responding to others' bids.

Second, the search engines effectively sell flows of perishable advertising services rather than storable objects: if there are no ads for a particular search term during some period of time, the "capacity" is wasted, much like in electricity markets. Of course, this environment is very different from a market for electricity: e.g., the marginal utility of advertisers from an additional click can be considered constant and hence can be reasonably well approximated by a single number, while the marginal benefit from electricity is rapidly diminishing, requiring bidders to submit entire demand curves; the market for electricity is two-sided, whereas sponsored search auctions are one-sided; etc. 
Finally, unlike other centralized markets, where it is usually clear how to measure what is being sold, there is no obvious definition of a "unit" of Internet advertisement. From the advertiser's perspective, the relevant unit is the cost of attracting a customer who makes a purchase. This corresponds most directly to a pricing model in which an advertiser pays only when a customer actually completes a transaction. From the search engine's perspective, the relevant unit is what it collects in revenues every time a user performs a search for a particular keyword. This corresponds to a pricing model in which an advertiser is charged every time its link is shown to a potential consumers. "Pay-per-click" is a middle ground between the two models: the advertiser pays every time a user clicks on the link. All three payment models are widely used on the Internet. ${ }^{1}$ The specific sector of Internet advertising that we study, sponsored search auctions, has converged to pay-per-click pricing after several years of evolution.

Since GSP evolved in the market for online advertising, its rules reflect the environment's unique characteristics. GSP insists that for each keyword, bidders submit a single bid - even though several different items are for sale: position 1 is very different from position 5 . The unusual onebid requirement makes sense in this setting: the value of being in each position is proportional to the number of clicks associated with that position. Consequently, even though the environment is multi-object, buyer valuations can be adequately represented by one-dimensional types. However, one bid per keyword is probably not sufficiently expressive to fully convey the preferences of the bidders: e.g., it does not allow for the possibility that the users who click on position 5 are somehow different from those who click on position 2 ; it does not allow for the possibility that advertisers care about the allocation of other positions, and so on. Nonetheless, these limitations are apparently not large enough to justify added complexity in the bidding language, and we will likewise ignore these possibilities in our model. One important possibility that we abstract away from is that ads from different advertisers have different probabilities of being clicked when placed in the same position. (These probabilities are known in the industry as "click-through rates", or CTRs.) Different search engines treat this possibility differently: Yahoo! ignores the CTRs, ranks the bidders purely in the order of decreasing bids, and charges the next-highest bidder's bid. In contrast, Google multiplies each advertiser's bid by its estimated CTR to compute its expected revenue, ranks the ads by these expected revenues, and then charges each bidder the smallest amount sufficient to exceed the expected revenue of the next bidder's bid times his estimated CTR. ${ }^{2}$ In our analysis, we assume that all bidders have identical CTRs, in which case Google's and Yahoo!'s mechanisms are identical. The analysis would remain largely the same if all advertisers' CTRs in any given position $x$ differed by a constant factor. ${ }^{3}$

\footnotetext{
${ }^{1}$ A prominent example of "pay-per-transaction," and even "pay-per-dollar of revenue" ("revenue sharing") is Amazon.com's Associates program, http://www.amazon.com/gp/browse.html?\&node=3435371 (accessed August 10, 2005). Under this program, a website that sends customers to Amazon.com receives a percentage of customers' purchases. "Pay-per-impression" advertising, in the form of banner ads, remains popular on major Internet portals, such as yahoo.com, msn.com, and aol.com.

${ }^{2}$ See https://adwords.google.com/select/faq/start.html (accessed August 15, 2005)

${ }^{3}$ The analysis would have to change considerably if there are specific advertiser-position effects. The magnitude of these specific advertiser-position effects is ultimately an empirical questions, and we do not have the kind of data that would allow us to answer it, but judging from the fact that the two major search engines effectively ignore it in
} 


\subsection{Evolution of Market Institutions}

The history of this sponsored search auctions is of interest as a case study of whether, how, and how quickly markets come to address their structural shortcomings. Many important mechanisms have recently been designed essentially from scratch, entirely replacing completely different historical allocation mechanisms: radio spectrum auctions, electricity auctions, and others. In contrast, reminiscent of the gradual evolution of medical residency match rules, sponsored search ad auctions have evolved in steps over time. In both medical residency and search advertising, flawed mechanisms were gradually replaced by increasingly superior designs. Notably, the Internet advertising market evolved much faster than the medical matching market. This may be due to the competitive pressures on mechanism designers present in the former but not in the latter, much lower costs of entry and experimentation, advances in the understanding of market mechanisms, and improved technology.

We proceed with a brief chronological review of the development of sponsored search mechanisms.

\subsubsection{Early Internet Advertising}

Beginning in 1994, Internet advertisements were largely sold on a per-impression basis. Advertisers paid flat fees to show their ads a fixed number of times (typically, one thousand showings, or "impressions"). Contracts were negotiated on a case-by-case basis, minimum contracts for advertising purchases were large (typically, a few thousand dollars per month), and entry was slow. ${ }^{4}$

\subsubsection{Generalized First-Price Auctions}

In 1997, Overture (then GoTo; now part of Yahoo!) introduced a completely new model of selling Internet advertising. In the original Overture auction design, each advertiser submitted a bid reporting the advertiser's willingness to pay on a per-click basis, for a particular keyword. The advertisers could now target their ads: instead of paying for a banner ad that would be shown to everyone visiting a website, advertisers could specify which keywords were relevant to their products, and how much each of those keywords (or, more precisely, a user clicking on their ad after looking for that keyword) was worth to them. Also, advertising was no longer sold per thousand impressions; rather, it was sold one click at a time. Every time a consumer clicked on a sponsored link, an advertiser's account was automatically billed the amount of the advertiser's most recent bid. The links to advertisers were arranged in descending order of bids, making highest bids the most prominent. The ease of use, the very low entry costs, and the transparency of the mechanism quickly led to the success of Overture's paid search platform as the advertising provider for major search engines including Yahoo! and MSN. However, the underlying auction mechanism itself was

their mechanisms (Yahoo! ignores CTRs altogether; Google computes an advertiser's estimated CTR conditional on the advertiser attaining the first position), we believe it to be small.

${ }^{4}$ See http://www.worldata.com/wdnet8/articles/the_history_of_Internet_Advertising.htm and http://www.zakon.org/robert/internet/timeline (both accessed August 10, 2005). 
far from perfect. In particular, Overture and advertisers quickly learned that the mechanism was unstable due to the dynamic nature of the environment.

Example. Suppose there are two slots on a page and three bidders. An ad in the first slot receives 200 clicks per hour, while the second slot gets 100. Bidders 1, 2, and 3 have values per click of $\$ 10, \$ 4$, and $\$ 2$, respectively. Suppose bidder 2 bids $\$ 2.01$, to guarantee that he gets a slot. Then bidder 1 will not want to bid more than $\$ 2.02$ - he does not need to pay more than that to get the top spot. But then bidder 2 will want to revise his bid to $\$ 2.03$ to get the top spot, bidder 1 will in turn raise his bid to $\$ 2.04$, and so on. Clearly, there is no pure strategy equilibrium in the one-shot version of the game, and so if bidders best respond to each other, they will want to revise their bids as often as possible. Figure 1 shows an example of this behavior on Overture.

\subsubsection{Generalized Second-Price Auctions}

Under the generalized first-price auction, the bidder who could react to its competitors' moves fastest had a substantial advantage. The mechanism therefore encouraged inefficient investments in gaming the system. It also created volatile prices that in turn caused allocative inefficiencies. Google addressed these problems when it introduced its own pay-per-click system, AdWords Select, in February 2002. Google also recognized that a bidder in position $i$ will never want to pay more than one bid increment above the bid of the advertiser in position $(i+1)$, and Google adopted this principle in its newly-designed generalized second price auction mechanism. In the simplest GSP auction, an advertiser in position $i$ pays a price per click equal to the bid of an advertiser in position $(i+1)$ plus a minimum increment (typically $\$ 0.01$ ). This second-price structure makes the market more user friendly and less susceptible to gaming.

Recognizing these advantages, Yahoo!/Overture also switched to GSP. Let us describe the version of GSP that it implemented. ${ }^{5}$ Every advertiser submits a bid. Advertisers are arranged on the page in descending order of their bids. The advertiser in the first position pays a price per click that equals the bid of the second advertiser plus an increment; the second advertiser pays the price offered by the third advertiser plus an increment; and so forth. ${ }^{6}$

Example (continued). Let us now consider the payments in the environment of the previous example under GSP mechanism. If all advertisers bid truthfully, then bids are $\$ 10, \$ 4, \$ 2$. Payments in GSP will be $\$ 4$ and $\$ 2 .{ }^{7}$ Truth-telling is indeed an equilibrium in this example, because no bidder can benefit by changing his bid. Note that total payments of bidders one and two are $\$ 800$ and $\$ 200$, respectively.

\footnotetext{
${ }^{5}$ We focus on Overture's implementation, because Google's system is somewhat more complex. However, it is straightforward to generalize our analysis to Google's mechanism.

${ }^{6}$ For this version of GSP to be efficient, it is necessary that the number of clicks received by an advertisement in a given position depends on the ad's position and not on the advertiser's identity. Recognizing that this assumption may not hold if some ads attract more clicks than others, Google adjusts effective bids based on ads' click-through rates. But under the assumption that all ads have the same click-through rates conditional on position, Google's and Yahoo!'s versions of GSP are identical. With modification for Google's adjustment procedure, our results for the Yahoo! version of GSP also hold for Google adjusted GSP.

${ }^{7}$ For convenience, we neglect the $\$ 0.01$ minimum increments.
} 


\subsection{Generalized Second-Price and VCG Auctions}

GSP looks similar to the Vickrey-Clarke-Groves mechanism, because both mechanisms set each agent's payments only based on the allocation and bids of other players, not based on that agent's own bid. In fact, Google's advertising materials explicitly refer to Vickrey and state that Google's "unique auction model uses Nobel Prize-winning economic theory to eliminate ... that feeling that you've paid too much". ${ }^{8}$ But GSP is not VCG. In particular, unlike the VCG auction, GSP does not have an equilibrium in dominant strategies, and truth-telling is generally not an equilibrium strategy in GSP. (See the example in Remark 3.) With only one slot, VCG and GSP would be identical. With several slots, the mechanisms are different: while GSP essentially charges bidder $i$ the bid of bidder $i+1$, VCG charges bidder $i$ the externality that he imposes on others, i.e., the decrease in the value of clicks received by other bidders because of $i$ 's presence.

Example (continued). Let us compute VCG payments for the example considered above. The second bidder's payment is $\$ 200$, as before. However, the payment of the first advertiser is now \$600: $\$ 200$ for the externality that he imposes on bidder 3 (by forcing him out of position 2) and $\$ 400$ for the externality that he imposes on bidder 2 (by moving him from position 1 to position 2 and thus causing him to lose $(200-100)=100$ clicks per hour). Note that in this example, revenues under VCG are lower than under GSP. As we will show later, if advertisers were to bid their true values under both mechanisms, revenues would always be higher under GSP.

\subsection{Assessing the Market's Development}

The chronology above suggests three major stages in the development of the sponsored search advertising market. First, ads were sold manually, slowly, in large batches, and on a cost-perimpression basis. Second, Overture implemented keyword-targeted per-click sales and began to streamline advertisement sales with some self-serve bidding interfaces, but with a highly unstable first-price mechanism. Next, Google implemented the generalized second price auction (GSP), which was subsequently adopted by Overture (Yahoo!).

Interestingly, Google and Yahoo! still use GSP, rather than VCG, which would reduce incentives for strategizing and make life easier for advertisers. We see several possible reasons for this. First, VCG is hard to explain to typical advertising buyers. Second, switching to VCG may entail substantial transition costs: VCG revenues are lower than GSP revenues for the same bids, and bidders might be slow to stop shading their bids. Third, the revenue consequences of switching to VCG are uncertain: even the strategic equivalence of second-price and English auctions under private values fails to hold in experiments (Kagel, Harstad, and Levin, 1987). And of course, simply implementing and testing a new system may be costly - imposing switching costs on advertisers as well as on search engines. Consequently, new entrants such as Ask Jeeves and Microsoft Network ${ }^{9}$ have a comparative advantage over the established players in implementing VCG.

\footnotetext{
${ }^{8}$ See https://www.google.com/adsense/afs.pdf (accessed August 10, 2005).

${ }^{9}$ See http://www.irconnect.com/askjinc/pages/news_releases.html?d=83045 and http://advertising.msn.com/searchadv/ (both accessed August 10, 2005).
} 


\section{The Rules of GSP}

Let us now formally describe the rules of a sponsored search auction. For a given keyword, there are $N$ objects (positions on the screen, where ads related to that keyword can be displayed) and $K$ bidders (advertisers). ${ }^{10}$ The (expected) number of clicks per period received by the bidder whose ad was placed in position $i$ is $\alpha_{i}$. The value per click to bidder $k$ is $s_{k}$. Bidders are risk-neutral, and bidder $k$ 's payoff from being in position $i$ is equal to $\alpha_{i} s_{k}$ minus his payments to the search engine. Note that these assumptions imply that the number of times a particular position is clicked does not depend on the ads in this and other positions, and also that an advertiser's value per click does not depend on the position in which its ad is displayed. Without loss of generality, positions are labeled in a descending order: for any $j$ and $k$ such that $j<k$, we have $\alpha_{j}>\alpha_{k}$.

We model the generalized second price auction (GSP) as follows. Suppose at some time $t$ a search engine user enters a given keyword, and, for each $k$, advertiser $k$ 's last bid submitted for this keyword prior to $t$ was $b_{k}$; if advertiser $k$ did not submit a bid, we set $b_{k}=0$. Let $b^{(j)}$ and $g(j)$ denote the bid and identity of the $j$-th highest bidder, respectively. If several advertisers submit the same bid, they are ordered randomly. ${ }^{11}$ The mechanism then allocates the top position to the bidder with the highest bid, $g(1)$, the second position to $g(2)$, and so on, down to position $\min \{N, K\}$. Note that each bidder gets at most one object. If a user clicks on a bidder's link, the bidder's payment per click is equal to the next bidder's bid. So bidder $g(i)$ 's total payment $p^{(i)}$ is equal to $\alpha_{i} b^{(i+1)}$ for $i \in\{1, \ldots, \min \{N, K\}\}$, and his payoff is equal to $\alpha_{i}\left(s^{(i)}-b^{(i+1)}\right)$. If there are at least as many positions as advertisers $(N \geq K)$, then the last bidder's payment $p^{(K)}$ is equal to zero. $^{12}$

It is also useful to describe explicitly the rules that the VCG mechanism would impose in this setting. The rules for allocating positions are the same as under GSP - the higher the bid, the better the position - but the payments are different. Each player's payment is equal to the negative externality that he imposes on others, assuming that bids are equal to values. Thus, the payment of the last bidder who gets allocated a spot is the same as under GSP: zero if $N \geq K ; \alpha_{N} b^{(N+1)}$ otherwise. For all other $i<\min \{N, K\}$, payment $p^{V}$ induced by VCG will be different from payment $p$ induced by GSP. Namely, $p^{V,(i)}=\left(\alpha_{i}-\alpha_{i+1}\right) b^{(i+1)}+p^{V,(i+1)}$.

In the following two sections, we will consider two alternative ways of completing the model: as a static game of complete information resembling a sealed-bid second-price auction and as a dynamic game of incomplete information resembling an English auction. Before moving on to these models, let us make a few observations about GSP and VCG.

Remark 1 If all advertisers were to bid the same amounts under the two mechanisms, then each advertiser's payment would be at least as large under GSP as under VCG.

\footnotetext{
${ }^{10}$ In actual sponsored search auctions at Google and Yahoo!, advertisers can also choose to place "broad match" bids that match searches that include a keyword along with additional search terms.

${ }^{11}$ The actual practice at Overture is to show equal bids according to the order in which the bidders placed their bids.

${ }^{12}$ Although we normalize the reserve price to zero, search engines charge the last bidder a non-zero reserve price.
} 
This is easy to show by induction on advertisers' payments, starting with the last advertiser who gets assigned a position. For $i=\min \{K, N\}, p^{(i)}=p^{V,(i)}=\alpha_{i} b^{(i+1)}$. For any $i<\min \{K, N\}$, $p^{V,(i)}-p^{V,(i+1)}=\left(\alpha_{i}-\alpha_{i+1}\right) b^{(i+1)} \leq \alpha_{i} b^{(i+1)}-\alpha_{i+1} b^{(i+2)}=p^{(i)}-p^{(i+1)}$.

Remark 2 Truth-telling is a dominant strategy under VCG.

This is a well-known property of the VCG mechanism.

Remark 3 Truth-telling is not a dominant strategy under GSP.

For instance, consider a slight modification of the example from Section 2. There are still three bidders, with values per click of $\$ 10, \$ 4$, and $\$ 2$, and two positions. However, the clickthrough rates of these positions are now almost the same: the first position receives 200 clicks per hour, and the second one gets 199. If all players bid truthfully, then bidder 1's payoff is equal to $(\$ 10-\$ 4) * 200=\$ 1200$. If, instead, he shades his bid and bids only $\$ 3$ per click, he will get the second position, and his payoff will be equal to $(\$ 10-\$ 2) * 199=\$ 1592>\$ 1200$.

\section{Static GSP and Locally Envy-Free Equilibria ${ }^{13}$}

Advertisers bidding on Yahoo! and Google can change their bids very frequently. We therefore think of these sponsored search auctions as continuous time or infinitely repeated games in which bidders originally have private information about their types, gradually learn the values of others, and can adjust their bids repeatedly. In principle, the sets of equilibria in such repeated games can be very large, with players potentially punishing each other for deviations. However, the strategies required to support such equilibria are usually quite complex, requiring precise knowledge of the environment and careful implementation. It may not be reasonable to expect the advertisers to be able to execute such strategies: they often manage thousands of keywords, and implementing sophisticated dynamic strategies for many keywords is likely to be prohibitively expensive and complex. In theory, advertisers could implement such strategies via automated robots, but at present they cannot do so: bidding software must first be authorized by the search engines, ${ }^{14}$ and search engines are unlikely to permit sophisticated strategies that would allow bidders to collude and reduce revenues. Indeed, leading software for bidding at sponsored search auctions does not allow advertisers to condition their bids on the prior behavior of specific competitors. ${ }^{15}$

We therefore focus on simple strategies that bidders can reasonably execute, and we study the rest points of the bidding process: if the vector of bids stabilizes, at what bids can it stabilize? We impose several assumptions and restrictions. First, we assume that all values are common knowledge: over time, bidders are likely to learn all relevant information about each other's values.

\footnotetext{
${ }^{13}$ After this research was completed, we found out that several of the results of this section were independently discovered by Hal Varian in unpublished work.

${ }^{14}$ See, e.g., http://www.content.overture.com/d/HKm/legal/zhhkrtc.jhtml (accessed August 18, 2005).

${ }^{15}$ See, e.g., Atlas Onepoint Rules-Based Bidding, http://www.atlasonepoint.com/products/bidmanager/rulesbased (accessed August 18, 2005), which lacks any bidding rule of this type.
} 
Second, since bids can be changed at any time, stable bids must be static best responses to each other - otherwise a bidder whose bid is not a best response would have an incentive to change it. Thus, we assume that the bids form an equilibrium in the static one-shot game of complete information. Third, what are the simple strategies that a bidder can use to increase his payoff, beyond simple best responses to the other players' bids?

One clear strategy is to try to force out the player who occupies the position immediately above. Suppose bidder $k$ bids $b_{k}$ and is assigned to position $i+1$ and bidder $k^{\prime}$ bids $b_{k^{\prime}}>b_{k}$ and is assigned to position $i$. Note that if $k$ raises his bid slightly, his own payoff does not change, but the payoff of the player above him decreases. Of course, player $k^{\prime}$ can retaliate, and the most he can do is to slightly underbid bidder $k$, effectively swapping places with him. If bidder $k$ is better off after such retaliation, he will indeed want to force player $k^{\prime}$ out, and the vector of bids will change. Thus, if the vector converges to a rest point, an advertiser in position $k$ should not want to "exchange" positions with the advertiser in position $k-1$. We call such vectors of bids "locally envy-free."

Definition 4 An equilibrium of the static game induced by GSP is locally envy-free if a player cannot improve his payoff by exchanging bids with the player ranked one position above him. More formally, in a locally envy-free equilibrium, for any $i \leq \min \{N, K\}$, we have $\alpha_{i} s_{g(i)}-p^{(i)} \geq$ $\alpha_{i-1} s_{g(i)}-p^{(i-1)}$.

Of course, it is possible that in the dynamic game bids change over time, depending on the player's strategies and information structure. However, as long as the restrictions are satisfied, if the dynamic game ever converges to a static vector of bids, that static equilibrium should correspond to a "locally envy-free" equilibrium of the static game $\Gamma$ induced by the GSP. Consequently, we view a locally envy-free equilibrium $\Gamma$ as a prediction regarding a rest point at which the vector of bids stabilizes. In this section, we study the set of locally envy-free equilibria.

We first show that the set of locally envy-free equilibria maps naturally to a set of stable assignments in a corresponding two-sided matching market. The idea that auctions and two-sided matching models are closely related is not new: it goes back to Crawford and Knoer (1981), Kelso and Crawford (1982), Leonard (1983), and Demange, Gale, and Sotomayor (1986), and has been studied in detail in a recent paper by Hatfield and Milgrom (2005). Note, however, that in our case the non-standard auction is very different from those in the above papers.

Our environment maps naturally into the most basic assignment model, studied first by Shapley and Shubik (1972). We view each position as an agent who is looking for a match with an advertiser. The value of an advertiser-position pair $(i, k)$ is equal to $\alpha_{k} s_{i}$. We call this assignment game $A$. The advertiser makes its payment $p_{i k}$ for the position, and the advertiser is left with $\alpha_{k} s_{i}-p_{i k}$. The following pair of lemmas shows that there is a natural mapping from the set of locally envy-free equilibria of GSP to the set of stable assignments. All proofs are in the Appendix.

Lemma 5 The outcome of any locally envy-free equilibrium of auction $\Gamma$ is a stable assignment.

Lemma 6 If the number of bidders is greater than the number of available positions, then any stable assignment is an outcome of a locally envy-free equilibrium of auction $\Gamma$. 
We will now construct a particular locally envy-free equilibrium of game $\Gamma$. This equilibrium has two important properties. First, in this equilibrium bidders' payments coincide with their payments in the dominant- strategy equilibrium of VCG. Second, this equilibrium is the worst locally envy-free equilibrium for the search engine, and it is the best locally envy-free equilibrium for the bidders. Consequently, the revenues of a search engine are (weakly) higher in any locally envy-free equilibrium of GSP than in the dominant-strategy equilibrium of VCG.

Consider the following strategy profile $B^{*}$. For each advertiser $i \in\{2, \ldots, \min \{N, K\}\}$, bid $b_{i}^{*}$ is equal to $\frac{p^{V,(i-1)}}{\alpha_{i-1}}$, where $p^{V,(j)}$ is the payment of bidder $j$ in the dominant-strategy equilibrium of VCG. Bid $b_{1}^{*}$ is equal to $s_{1} \cdot{ }^{16}$

Theorem 7 Strategy profile $B^{*}$ is a locally envy-free equilibrium of game $\Gamma$. In this equilibrium, each bidder's position and payment is equal to those in the dominant-strategy equilibrium of the game induced by VCG. In any other locally envy-free equilibrium of game $\Gamma$, the total revenue of the seller is at least as high as in $B^{*}$.

To prove Theorem 7 , we first note that payments under strategy profile $B^{*}$ coincide with VCG payments and check that $B^{*}$ is indeed a locally-envy free equilibrium. This follows from the fact that, by construction, each bidder is indifferent between remaining in his positions and swapping with the bidder one position above him. Next, from Lemma 5 we know that every locally envy-free equilibrium corresponds to a stable assignment. Combining this with the fact ${ }^{17}$ that in any stable assignment the payments of bidders must be at least as high as VCG payments completes the proof.

\section{Main Result: GSP and the Generalized English Auction}

In this section we introduce the generalized English auction that corresponds to the generalized second price auction. The generalized English auction is a useful metaphor for predicting the behavior of advertisers in GSP auctions in a dynamic environment. In the model analyzed in the previous section, we assume that bidders have converged to a long-run steady state, have learned each other's values, and no longer have incentives to change their bids. But how do they converge to such a situation?

In this section we show that, in fact, there are simple strategies that can quickly lead to such an outcome: each advertiser starts bidding at zero and keeps increasing his bid as long as he finds it profitable to do so. This simple myopic procedure leads to an equilibrium outcome in which bidders have no incentives to change their bids. Remarkably, the outcome is the same as the worst (for the search engine) locally-envy free equilibrium of the static GSP: all players get VCG payoffs.

We model this procedure through an analogue of the standard English auction. The generalized English Auction entails a clock showing the current price (that continuously increases over time). A player's bid is the price on the clock at the time when he drops out. The auction is over when

\footnotetext{
${ }^{16}$ This bid does not affect any bidder's payment and can be set equal to any value greater than $b_{2}^{*}$.

${ }^{17}$ Leonard (1983) states this fact for general assignment problems. For completeness, we include a short independent proof that relies on the specifics of our particular model.
} 
the next-to-last advertiser drops out. The ad of the last remaining advertiser is placed in the best advertisement position, and this advertiser's payment per click is equal to the price at which the next-to-last advertiser dropped out. The ad of the next-to-last advertiser is placed second, and its payment per click is equal to the third-highest advertiser's bid, and so on. In other words, the vector of bids obtained in the generalized English auction is used to allocate the objects and compute the prices according to the rules of GSP, just as the rules of the second price auction can be used to compute the payments in the English auction. ${ }^{18}$ The following theorem shows that this game has a unique perfect Bayesian equilibrium with strategies continuous in bidders' valuations. ${ }^{19}$

For concreteness, assume that there are $N \geq 2$ slots and $K=N+1$ advertisers. (Cases in which $K \neq N+1$ require only minor modifications in the proof.) Click-through rates $\alpha_{n}$ are commonly known, with $\alpha_{N+1} \equiv 0$. Bidders' per-click valuations $s_{i}$ are drawn from a continuous distribution $F(\cdot)$ on $[0 ;+\infty)$ with a continuous density function $f(\cdot)$ that is positive everywhere on $(0,+\infty)$. Each advertiser knows his valuation and the distribution of other advertisers' valuations.

The strategy of an advertiser assigns the choice of dropping out or not for any history of the game, given that the advertiser has not previously dropped out. In other words, the strategy can be represented as a function $p_{i}\left(k, h, s_{i}\right)$, where $s_{i}$ is the value per click of bidder $i, p_{i}$ is the price at which he drops out, $k$ is the number of bidders remaining (including bidder $i$ ), and $h=\left(b_{k+1}, \ldots, b_{K}\right)$ is the history of prices at which bidders $K, K-1, \ldots, k+1$ have dropped out. (As a result, the price that bidder $i$ would have to pay per click if he dropped out next is equal to $b_{k+1}$, unless the history is empty, in which case we say that $b_{k+1} \equiv 0$.)

Theorem 8 In the unique perfect Bayesian equilibrium of the generalized English auction with strategies continuous in $s_{i}$, an advertiser with value $s_{i}$ drops out at price $p_{i}\left(k, h, s_{i}\right)=s_{i}-\frac{\alpha_{k}}{\alpha_{k-1}}\left(s_{i}-\right.$ $\left.b_{k+1}\right)$. In this equilibrium, each advertiser's resulting position and payoff are the same as in the dominant-strategy equilibrium of the game induced by VCG. This equilibrium is ex post: the strategy of each bidder is a best response to other bidders' strategies regardless of their realized values.

The intuition of the proof is as follows. First, with $k$ players remaining and the next highest bid equal to $b_{k+1}$, it is a dominated strategy for a player with value $s$ to drop out before price $p$ reaches the level at which he is indifferent between getting position $k$ and paying $b_{k+1}$ per click and getting position $k-1$ and paying $p$ per click. Next, if for some set of types it is not optimal to drop out at this "borderline" price level, we can consider the lowest such type, and then once the clock reaches this price level, a player of this type will know that he has the lowest per-click value of the

\footnotetext{
${ }^{18}$ If several players drop out simultaneously, one of them is chosen randomly. Whenever a player drops out, the clock is stopped, and other players are also allowed to drop out; again, if several ones want to drop out, one is chosen randomly. If several players end up dropping at the same price, the first one to drop out is placed in the lowest position of the still available ones, the next one - to the position right above that, and so on.

${ }^{19}$ Without this restriction, multiple equilibria exist, even in the simplest English auction with two bidders and one object. For example, suppose there is one object for sale and two bidders with independent private values for this object distributed exponentially on $[0, \infty)$. Consider the following pair of strategies. If a bidder's value is in the interval $[0,1]$ or in the interval $[2, \infty)$, he drops out when the clock reaches his value. If bidder 1 's value is in the interval $(1,2)$, he drops out at 1 , and if bidder 2's value is in the interval $(1,2)$, he drops out at 2 . This pair of strategies, together with appropriate beliefs, forms a perfect Bayesian equilibrium.
} 
remaining players. But then he will also know that the other remaining players will only drop out at price levels at which he will find it unprofitable to compete with them for the higher positions.

The result of Theorem 8 resembles the classic result on the equivalence of the English auction and the second price sealed-bid auction under private values (Vickrey, 1961). Note, however, that the intuition is very different: Vickrey's result follows simply from the existence of equilibria in dominant strategies, whereas in our case such strategies do not exist, and bids do depend on other player's bids. Also, our result is very different from the revenue equivalence theorem: payoffs in the generalized English auction coincide with VCG payments for all realizations of values, not only in expectation; bidders can be asymmetric; distributions of valuations need not be commonly known.

The equilibrium described in Theorem 8 is an ex post equilibrium. As long as all bidders other than bidder $i$ follow the equilibrium strategy described in Theorem 8, it is a best response for bidder $i$ to follow his equilibrium strategy, for any realization of other bidders' values. Thus, the outcome implemented by this mechanism depends only on the realization of bidders' values and does not depend on bidders' beliefs about each others' types.

Obviously, any dominant strategy solvable game has an ex post equilibrium. However, the generalized English auction is not dominant strategy solvable. This combination of properties is quite striking: the equilibrium is unique and efficient, the strategy of each bidder does not depend on the distribution of other bidders' values, yet bidders do not have dominant strategies. ${ }^{20,21}$ The generalized English auction is a particularly interesting example, because it can be viewed as a model of a mechanism that has "emerged in the wild."

\section{Conclusion}

We investigate a new mechanism that we call the generalized second price auction. GSP is tailored to the unique features of the market for Internet advertisements. As far as we know, this mechanism was first used in 2002. As of September 2005, the annual revenues from GSP auctions were in the billions of dollars.

GSP looks similar to the VCG mechanism, because just like in the standard second price auction, the payment of a bidder does not directly depend on his bid. Although GSP looks similar to VCG, its properties are very different, and equilibrium behavior is far from straightforward. In particular, unlike the VCG mechanism, GSP generally does not have an equilibrium in dominant strategies, and truth-telling is not an equilibrium of GSP. We show that the generalized English auction that corresponds to the generalized second-price auction has a unique equilibrium.

\footnotetext{
${ }^{20}$ Bergemann and Morris (2004) show that an outcome implementable by robust mechanisms must be implementable in dominant strategies. Indeed, the outcome implemented by the generalized English auction can be implemented in dominant strategies by VCG mechanism; however, VCG is not the mechanism that is used in practice.

${ }^{21}$ Jehiel and Moldovanu (2001) and Jehiel et al. (2005) show that generically, any efficient choice function is not Bayes-Nash implementable and any non-trivial choice function is not ex post implementable, if values are interdependent and signals are multi-dimensional. Of course, in our model values are private, and, crucially, signals are single-dimensional, even though multiple different objects are for sale. This makes efficient ex post implementation feasible. For other examples of mechanisms that allocate multiple different objects to bidders with single-dimensional types see Moldovanu and Sela (2001), Kittsteiner and Moldovanu (2005), and references in those papers.
} 
This equilibrium has some notable properties. The bid functions have explicit analytic formulas, which, combined with equilibrium uniqueness, makes our results a useful starting point for empirical analysis. Moreover, these functions do not depend on bidders' beliefs about each others' types: the outcome of the auction only depends on the realizations of bidders' values. Hence, the generalized English auction is a robust mechanism, even though bidders do not have dominant strategies. This is one of the very few mechanisms encountered in practice that are not dominant strategy solvable yet robust. It is particularly interesting that a mechanism with such notable features in theory and such enormous popularity in practice developed as a result of evolution of inefficient market institutions, which were gradually replaced by increasingly superior designs.

\section{References}

[1] Bergemann, D., and S. Morris (2004), "Robust Mechanism Design," Econometrica, forthcoming.

[2] Crawford, V., and E. Knoer (1981), "Job Matching with Heterogeneous Firms and Workers," Econometrica, 49, 437-450.

[3] Demange, G., D. Gale, and M. Sotomayor (1986), "Multi-Item Auctions," Journal of Political Economy, $94,863-872$.

[4] Hatfield, J., and P. Milgrom (2005), "Auctions, Matching and the Law of Aggregate Demand," American Economic Review, forthcoming.

[5] Kagel, J., R. Harstad, and D. Levin (1987), "Information Impact and Allocation Rules in Auctions with Affiliated Private Values: A Laboratory Study," Econometrica, 55, 1275-1304.

[6] Kittsteiner, T., and B. Moldovanu (2005), "Priority Auctions and Queue Disciplines that Depend on Processing Time," Management Science, 51, 236-248.

[7] Kelso, A., and V. Crawford (1982), "Job Matching, Coalition Formation, and Gross Substitutes," Econometrica, 50, 1483-1504.

[8] Jehiel, P., and B. Moldovanu (2001), "Efficient Design with Interdependent Valuations," Econometrica, $69,1237-1259$.

[9] Jehiel, P., B. Moldovanu, M. Meyer-ter-Vehn, and W. Zame (2005), "The Limits of Ex-Post Implementation," working paper.

[10] Leonard, H., (1983), "Elicitation of Honest Preferences for the Assignment of Individuals to Positions," Journal of Political Economy, 91, 461-478.

[11] Moldovanu, B., and A. Sela (2001), "The Optimal Allocation of Prizes in Contests," American Economic Review, 91, 542-558.

[12] Shapley, L., and M. Shubik (1972), "The Assignment Game I: The Core," International Journal of Game Theory, 1, 111-130.

[13] Vickrey, W., (1961), "Counterspeculation, Auctions, and Competitive Sealed Tenders," Journal of Finance, 16, 8-27. 


\section{Appendix}

\section{Proof of Lemma 5}

By definition, in any locally envy-free equilibrium outcome, no player can profitably re-match with the position assigned to the player right above him. Also, no player (a) can profitably re-match with a position assigned to a player below him $(b)$ - if such a profitable re-matching existed, player $a$ would find it profitable to slightly undercut player $b$ in game $\Gamma$ and get $b$ 's position and payment. But this would contradict the assumption that we are in equilibrium. ${ }^{22}$

Hence, we only need to show that no player can profitably re-match with the position assigned to a player more than one spot above him. First, note that in any locally envy-free equilibrium, the resulting matching must be assortative, i.e., for any $i$, the player assigned to position $i$ has a higher per-click valuation than the player assigned to position $i+1$, and therefore, player 1 must be assigned to the top position, player 2-to the second-highest position, and so on.

Indeed, let $s_{i}$ and $s_{i+1}$ denote the values of players assigned to positions $i$ and $i+1$. Equilibrium restrictions imply that $\alpha_{i} s_{i}-p_{i} \geq \alpha_{i+1} s_{i}-p_{i+1}$ (nobody wants to move one position down), and local envy-freeness implies that $\alpha_{i+1} s_{i+1}-p_{i+1} \geq \alpha_{i} s_{i+1}-p_{i}$ (nobody wants to move one position up). Manipulating the above inequalities yields $\alpha_{i} s_{i}-\alpha_{i} s_{i+1}+\alpha_{i+1} s_{i+1} \geq \alpha_{i+1} s_{i}$, thus $\left(\alpha_{i}-\alpha_{i+1}\right) s_{i} \geq\left(\alpha_{i}-\alpha_{i+1}\right) s_{i+1}$. Since $\alpha_{i}>\alpha_{i+1}$, we have $s_{i} \geq s_{i+1}$, and hence the locally envy-free equilibrium outcome must be an assortative match.

Now, let us show that no player can profitably re-match with the position assigned to a player more than one spot above him. Suppose bidder $k$ is considering re-matching with position $m<k-1$. Since the equilibrium is locally envy-free, we have

$$
\begin{aligned}
\alpha_{k} s_{k}-p_{k} & \geq \alpha_{k-1} s_{k}-p_{k-1} \\
\alpha_{k-1} s_{k-1}-p_{k-1} & \geq \alpha_{k-2} s_{k-1}-p_{k-2} \\
\vdots & \\
\alpha_{m+1} s_{m+1}-p_{m+1} & \geq \alpha_{m} s_{m+1}-p_{m} .
\end{aligned}
$$

Since $\alpha_{j}>\alpha_{j+1}$ for any $j$, and $s_{j}>s_{k}$ for any $j<k$, the above inequalities remain valid after replacing $s_{j}$ with $s_{k}$. Doing that, then adding all inequalities up, and canceling out the redundant elements, we get $\alpha_{k} s_{k}-p_{k} \geq \alpha_{m} s_{k}-p_{m}$. But that implies that advertiser $k$ cannot re-match profitably with position $m$, and we are done.

\section{Proof of Lemma 6}

Take a stable assignment. By a result of Shapley and Shubik (1972), this assignment must be efficient, and hence assortative, and so we can talk about advertiser $i$ being matched with position

\footnotetext{
${ }^{22}$ This argument relies on the fact that in equilibrium, no two (or more) players bid the same amount, which is straightforward to prove: since all players' per-click values are different, and all ties are broken randomly with equal probabilities, at least one of such players would find it profitable to bid slightly higher or slightly lower.
} 
$i$, with associated payment $p_{i}$.

Let us construct a locally envy-free equilibrium with the corresponding outcome. Let $b_{1}=s_{1}$ and $b_{i}=\frac{p_{i-1}}{\alpha_{i-1}}$ for $i>1$. Let us show that this set of strategies is a locally envy-free equilibrium. First, note that for any $i, b_{i}>b_{i+1}$ (because otherwise we would have, for some $i, \frac{p_{i-1}}{\alpha_{i-1}} \leq \frac{p_{i}}{\alpha_{i}} \Rightarrow$ $s_{i}-\frac{p_{i-1}}{\alpha_{i-1}} \geq s_{i}-\frac{p_{i}}{\alpha_{i}} \Rightarrow \alpha_{i-1}\left(s_{i}-p_{i-1}\right)>\alpha_{i}\left(s_{i}-p_{i}\right)$, which would imply that player $i$ could rematch profitably). Therefore, position allocations and payments resulting from this strategy profile will coincide with those in the original stable assignment. To see that this strategy profile is an equilibrium, note that deviating and moving to a different position in this strategy profile is at most as profitable for any player as re-matching with the corresponding position in the assignment game. To see that this equilibrium is locally envy-free, note that the payoff from swapping with the bidder above is exactly equal to the payoff from re-matching with that player's position in the assignment game.

\section{Proof of Theorem 7}

First, we need to check that the order of the bids is preserved, i.e., $b_{i}^{*}>b_{i+1}^{*}$ for any $i<\min \{N, K\}$. For $i \geq 2$, this is equivalent to

$$
\begin{aligned}
\frac{p^{V,(i-1)}}{\alpha_{i-1}} & >\frac{p^{V,(i)}}{\alpha_{i}} \\
& \Uparrow \\
\frac{\left(\alpha_{i-1}-\alpha_{i}\right) s_{i}+p^{V,(i)}}{\alpha_{i-1}} & >\frac{p^{V,(i)}}{\alpha_{i}} \\
& \Uparrow \\
\alpha_{i}\left(\alpha_{i-1}-\alpha_{i}\right) s_{i} & >\left(\alpha_{i-1}-\alpha_{i}\right) p^{V,(i)} \\
& \mathbb{} \\
\alpha_{i} s_{i} & >p^{V,(i)} .
\end{aligned}
$$

For $i=1, b_{i}^{*}>b_{i+1}^{*}$ is equivalent to

$$
\begin{aligned}
s_{1} & >\frac{p^{V,(1)}}{\alpha_{1}} \\
& \mathbb{} \\
\alpha_{1} s_{1} & >p^{V,(1)} .
\end{aligned}
$$

To see that for any $i, \alpha_{i} s_{i}>p^{V,(i)}$, note first that in the game induced by VCG, each player can guarantee himself the payoff of at least zero (by bidding zero), and hence in any equilibrium his 
payoff from clicks is at least as high as his payment. To prove that the inequality is strict, note that if player $i$ 's value per click were slightly lower, e.g., $s_{i}-\Delta$ instead of $s_{i}, \Delta<s_{i}-s_{i+1}$, then his payment in the truth-telling equilibrium would still be the same (because it does not depend on his own bid, given the allocation of positions), and so $p^{V,(i)} \leq \alpha_{i}\left(s_{i}-\Delta\right)<\alpha_{i} s_{i}$. Thus, for any $i$, $b_{i}^{*}>b_{i+1}^{*}$, and therefore each bidder's position is the same as in the truthful equilibrium of VCG. Therefore, by construction, payments are also the same.

Next, to see that no bidder $i$ can benefit by bidding less than $b_{i}^{*}$, suppose that he bids an amount $b^{\prime}<b_{i}^{*}$ that puts him in position $i^{\prime}>i$. Then, by construction, his payment will be equal to the amount that he would need to pay to be in position $i^{\prime}$ under VCG, provided that other players bid truthfully. But truthful bidding is an equilibrium under VCG, and so such deviation cannot be profitable there - hence, it cannot be profitable in strategy profile $B^{*}$ of game $\Gamma$ either.

To see that no bidder $i$ can benefit by bidding more than $b_{i}^{*}$, suppose that he bids an amount $b^{\prime}>b_{i}^{*}$ that puts him in position $i^{\prime}<i$. Then the net payoff from this deviation is equal to $\left(\alpha_{i^{\prime}}-\alpha_{i}\right) s_{i}-\left(\alpha_{i^{\prime}} b_{i^{\prime}}^{*}-\alpha_{i} b_{i+1}\right)<\left(\alpha_{i^{\prime}}-\alpha_{i}\right) s_{i}-\left(\alpha_{i^{\prime}} b_{i^{\prime}+1}^{*}-\alpha_{i} b_{i+1}\right)=\sum_{j=i^{\prime}}^{i-1}\left(\alpha_{j}-\alpha_{j+1}\right) s_{i}-\sum_{j=i^{\prime}}^{i-1}\left(p^{V,(j)}-\right.$ $\left.p^{V,(j+1)}\right)=\sum_{j=i^{\prime}}^{i-1}\left(\alpha_{j}-\alpha_{j+1}\right) s_{i}-\sum_{j=i^{\prime}}^{i-1}\left(\alpha_{j}-\alpha_{j+1}\right) s_{j+1}$. But since $s_{i} \leq s_{j+1}$ for any $j<i$, the last expression is less than or equal to zero, and hence the deviation is not profitable.

To check that this equilibrium is locally envy-free, note that if bidder $i$ swapped his bids with bidder $i-1$, his payoff would change by $\left(\alpha_{i-1}-\alpha_{i}\right) s_{i}-\left(\alpha_{i-1} b_{i}^{*}-\alpha_{i} b_{i+1}\right)=\left(\alpha_{i-1}-\alpha_{i}\right) s_{i}-\left(p^{V,(i-1)}-\right.$ $\left.p^{V,(i)}\right)=\left(\alpha_{i-1}-\alpha_{i}\right) s_{i}-\left(p^{V,(i-1)}-p^{V,(i)}\right)=\left(\alpha_{i-1}-\alpha_{i}\right) s_{i}-\left(\alpha_{i-1}-\alpha_{i}\right) s_{i}=0$. In other words, each bidder is indifferent between his actual payoff and his payoff after swapping bids with the bidder above, and hence the equilibrium is locally envy-free.

Let us now show that $B^{*}$ is the best locally-envy free equilibrium for the bidders and the worst locally envy-free equilibrium for the search engine. A standard result of matching theory states that there exists an assignment that is the best stable assignment for all advertisers, and the worst stable assignment for all positions. A stable assignment is characterized by a vector of payments $p=\left(p_{1}, \ldots, p_{K}\right)$. Let $p^{V}=\left(p_{1}^{V}, \ldots, p_{K}^{V}\right)$ be the set of dominant-strategy VCG payments, i.e., the set of payments in equilibrium $B^{*}$ of game $\Gamma$.

In any stable assignment, $p_{K}$ must be at least as high as $\alpha_{K} s_{K-1}$, since otherwise advertiser $K-1$ would find it profitable to match with position $K$. On the other hand, $p_{K}^{V}=\alpha_{K} s_{K-1}$, and hence in the buyer-optimal stable assignment, $p_{K}=p_{K}^{V}$.

Next, in any stable assignment, it must be the case that $p_{K-1}-p_{K} \geq\left(\alpha_{K-1}-\alpha_{K}\right) s_{K}-$ otherwise, advertiser $K$ would find it profitable to re-match with position $K-1$. Hence, $p_{K-1} \geq$ $\left(\alpha_{K-1}-\alpha_{K}\right) s_{K}+p_{K} \geq\left(\alpha_{K-1}-\alpha_{K}\right) s_{K}+p_{K}^{V}=p_{K-1}^{V}$, and so in the buyer-optimal stable assignment, $p_{K-1}=p_{K-1}^{V}$.

Proceeding by induction, we get $p_{k}=p_{k}^{V}$ for any $k \leq K$ in the buyer-optimal (and therefore seller-pessimal) stable assignment, and so in any locally envy-free equilibrium of game $\Gamma$, the total revenue of the seller is at least as high as $\sum_{k=1}^{K} p_{k}^{V}$. 


\section{Proof of Theorem 8}

First, note that in equilibrium, for any player $i$, any history $h$, and any number of remaining players $k$, the drop-out price $p_{i}\left(k, h, s_{i}\right)$ tends to infinity as $s_{i}$ tends to infinity. (Otherwise there would exist a player for whom it was optimal to deviate from his strategy and stay longer, for a sufficiently high value s.) Next, take any equilibrium of the generalized English auction. Note that if in this equilibrium $p_{i}\left(k, h, s_{i}\right)>p_{i}\left(k, h, s_{i}^{\prime}\right)$ for some $k, h, i$, and types $s_{i}<s_{i}^{\prime}$, then it has to be the case that both types $s_{i}$ and $s_{i}^{\prime}$ are indifferent between dropping out at $p_{i}\left(k, h, s_{i}\right)$ and $p_{i}\left(k, h, s_{i}^{\prime}\right)$. (Otherwise one of them would be able to increase his payoff by mimicking the other.) Consequently, we can "swap" such players' strategies, and therefore there exists an "observationally equivalent" equilibrium in which strategies are nondecreasing in types; also, they are still continuous in own values. Consider this equilibrium profile of strategies $p_{i}\left(k, h, s_{i}\right)$.

Let $q\left(k, b_{k+1}, s\right)$ be such a price that a player with value $s$ is indifferent between getting position $k$ at price $b_{k+1}$ and position $k-1$ at price $q\left(k, b_{k+1}, s\right)$. That is,

$$
\begin{aligned}
\alpha_{k-1}\left(s-q\left(k, b_{k+1}, s\right)\right) & =\alpha_{k}\left(s-b_{k+1}\right) \\
& \Uparrow \\
q\left(k, b_{k+1}, s\right) & =s-\frac{\alpha_{k}}{\alpha_{k-1}}\left(s-b_{k+1}\right) .
\end{aligned}
$$

Slightly abusing notation, let $q(k, h, s)=q\left(k, b_{k+1}, s\right)$, where $b_{k+1}$ is the last bid at which a player dropped out in history $h$. (This player received position $k+1$. If history $h$ is empty, we set $b_{k+1}=0$.) We will now show that for any $i, k, h$, and $s_{i}, p_{i}\left(k, h, s_{i}\right)=q\left(k, h, s_{i}\right)$.

Suppose that is not the case, and take the largest $k$ for which there exist such history $h$ (with the last player dropping out at $b_{k+1}$ ), player $i$, and type $s_{i}$ (surviving with positive probability on the equilibrium path) that $p_{i}\left(k, h, s_{i}\right) \neq q\left(k, h, s_{i}\right)$. Since by assumption, all strategies up to this stage were $p_{i}(\cdot, \cdot, \cdot)=q(\cdot, \cdot, \cdot)$, we know that there exists a value $s_{\min } \geq b_{k+1}$, such that all players with values less than $s_{\text {min }}$ have dropped out, and all players with values greater than $s_{\text {min }}$ are still in the auction.

Step 1. Suppose for some type $s \geq s_{\min }, p_{\max }(k, h, s) \equiv \max _{i} p_{i}(k, h, s)>q(k, h, s)$. Let $s_{0}$ be the smallest type, and let $i$ be the corresponding player, such that $p_{i}\left(k, h, s_{0}\right)=p_{\max }(k, h, s)$. Without loss of generality, we can assume that there is a positive mass of types of other players dropping out at or before $p_{i}\left(k, h, s_{0}\right) \cdot{ }^{23}$

Step 1(a). Suppose first that there is a positive mass of types of other players dropping out at $p_{i}\left(k, h, s_{0}\right)=p_{\max }(k, h, s)$. That implies that with positive probability, player $i$ of type $s_{0}$ will remain in the subgame following the drop-out of some other player at $p_{i}\left(k, h, s_{0}\right)$ (since ties are broken randomly). Let us show that in this subgame, player $i$ of type $s_{0}$ will be the first player to

\footnotetext{
${ }^{23}$ Otherwise, we have $\forall j \neq i, p_{j}\left(k, h, s_{0}\right) \leq p_{i}(k, h, s) \leq p_{\max }(k, h, s)=p_{i}\left(k, h, s_{0}\right) \Rightarrow \forall j \neq i, p_{j}\left(k, h, s_{0}\right)=$ $p_{i}\left(k, h, s_{0}\right)$ and $\forall s^{\prime}>s_{0}, p_{j}\left(k, h, s^{\prime}\right)>p_{i}\left(k, h, s_{0}\right)$. But we also have $p_{i}\left(k, h, s_{0}\right)=p_{\max }(k, h, s)>q(k, h, s) \geq$ $q\left(k, h, s_{0}\right)$, and so for some $s^{\prime}>s_{0}$ we have $p_{\max }\left(k, h, s^{\prime}\right)>q\left(k, h, s^{\prime}\right)$ and $p_{\max }\left(k, h, s^{\prime}\right)>p_{\max }(k, h, s)$. We can then consider $s_{0}^{\prime}$ in place of $s_{0}$, where $s_{0}^{\prime}$ is the smallest type, and $i^{\prime}$ is the corresponding player, such that $p_{i^{\prime}}\left(k, h, s_{0}^{\prime}\right)=p_{\max }\left(k, h, s^{\prime}\right)$. There is a positive mass of types of other players dropping out before $p_{i^{\prime}}\left(k, h, s_{0}^{\prime}\right)$.
} 
drop out with probability 1 . Suppose that is not the case, and let $l<k-1$ be the smallest number such that he gets position $l$ with positive probability.

Consider any continuation of history $h, h_{l+2}$, such that the last player to drop out in that history gets position $l+2$ and drops out at price $b_{l+2}$, player $i$ of type $s_{0}$ is one of the remaining $l+1$ players, there is a positive probability that player $i$ gets position $l$ in the continuation subgame following history $h_{l+2}$, and there is zero probability that player $i$ gets position $m$ for any $m<l$. Note that $s_{0} \geq b_{l+2}$ - otherwise it would have been optimal for player $i$ to drop out earlier. Consider $p_{i}\left(l+1, h_{l+2}, s_{0}\right)$. There must be a positive mass of types of other players who drop out no later than $p_{i}\left(l+1, h_{l+2}, s_{0}\right)$. Take the highest such type, $s^{\prime}$, and the corresponding player $j$. It has to be the case that $s^{\prime}>s_{0} \geq b_{l+2}$. It also has to be the case that $q\left(l+1, h_{l+2}, s^{\prime}\right)$ is less than or equal to $p_{i}\left(l+1, h_{l+2}, s_{0}\right)$. (Otherwise player $j$ with value $s^{\prime}$ would be playing a strategy weakly dominated by dropping out at $q\left(l+1, h_{l+2}, s^{\prime}\right)$, with a positive probability of earning strictly less than he would have earned if he waited until that price level.) Therefore, $p_{i}\left(l+1, h_{l+2}, s_{0}\right) \geq q\left(l+1, h_{l+2}, s^{\prime}\right)>q\left(l+1, h_{l+2}, s_{0}\right) \geq b_{l+2}$. Let us show that it would be strictly better for player $i$ with type $s_{0}$ to drop out at $q\left(l+1, h_{l+2}, s_{0}\right)$ instead of waiting until $p_{i}\left(l+1, h_{l+2}, s_{0}\right)$. Indeed, if nobody else drops out in between, or someone drops out before $q\left(l+1, h_{l+2}, s_{0}\right)$, these strategies would result in identical payoffs. Otherwise payoffs are different, and this happens with positive probability. Under the former strategy, player $i$ earns

$$
\alpha_{l+1}\left(s_{0}-b_{l+2}\right) .
$$

Under the latter strategy, he earns

$$
\alpha_{l}\left(s_{0}-b_{l+1}\right),
$$

where $b_{l+1}$ is the price at which somebody else dropped out. (The probability of getting a spot $m<l$ is zero by construction.) With probability $1, b_{l+1} \geq q\left(l+1, h_{l+2}, s_{0}\right)$, and with positive probability $b_{l+1}>q\left(l+1, h_{l+2}, s_{0}\right)$, so the expected payoff from waiting until $p_{i}\left(l+1, h_{l+2}, s_{0}\right)$ is strictly less than the expected payoff from dropping out at $q\left(l+1, h_{l+2}, s_{0}\right): E\left[\alpha_{l}\left(s_{0}-b_{l+1}\right)\right]<$ $\alpha_{l}\left(s_{0}-q\left(l+1, h_{l+2}, s_{0}\right)\right)=\alpha_{l}\left(s_{0}-\left(s_{0}-\frac{\alpha_{l+1}}{\alpha_{l}}\left(s_{0}-b_{l+2}\right)\right)\right)=\alpha_{l+1}\left(s_{0}-b_{l+2}\right)$.

Therefore, in the subgame following the drop-out of some other player at $p_{i}\left(k, h, s_{0}\right)$, player $i$ of type $s_{0}$ gets position $k-1$ with probability 1 , and therefore his payoff is $\alpha_{k-1}\left(s_{0}-p_{i}\left(k, h, s_{0}\right)\right)$. Now suppose player $i$ dropped out at a price $p_{i}\left(k, h, s_{0}\right)-\epsilon$ instead of waiting until $p_{i}\left(k, h, s_{0}\right)$. If somebody else drops out before $p_{i}\left(k, h, s_{0}\right)-\epsilon$ or after $p_{i}\left(k, h, s_{0}\right)$, or drops out at $p_{i}\left(k, h, s_{0}\right)$ but player $i$ is chosen to drop out first, then these two strategies result in identical payoffs. The probability that somebody drops out in the interval $\left(p_{i}\left(k, h, s_{0}\right)-\epsilon, p_{i}\left(k, h, s_{0}\right)\right)$ goes to zero as $\epsilon$ goes to zero, and the possible difference in the payoffs is finite, so the difference in payoffs due to this contingency goes to zero as $\epsilon$ goes to zero. Finally, there is a positive probability that somebody else drops out at $p_{i}\left(k, h, s_{0}\right)$ and is chosen to drop out first. If player $i$ drops out before that, at $p_{i}\left(k, h, s_{0}\right)-\epsilon$, his payoff is $\alpha_{k}\left(s_{0}-b_{k+1}\right)$. If he waits until $p_{i}\left(k, h, s_{0}\right)$, we know that in the subsequent subgame his payoff is $\alpha_{k-1}\left(s_{0}-p_{i}\left(k, h, s_{0}\right)\right)<\alpha_{k-1}\left(s_{0}-q\left(k, h, s_{0}\right)\right)=\alpha_{k}\left(s_{0}-b_{k+1}\right)$. 
Therefore, for a sufficiently small $\epsilon$, it is strictly better for player $i$ with value $s_{0}$ to drop out at $p_{i}\left(k, h, s_{0}\right)-\epsilon$ instead of waiting until $p_{i}\left(k, h, s_{0}\right)$, which contradicts the assumption that $\left\{p_{j}(\cdot, \cdot, \cdot)\right\}$ is an equilibrium.

Step 1(b). Now suppose there is mass zero of types of other players dropping out at $p_{i}\left(k, h, s_{0}\right)=$ $p_{\text {max }}(k, h, s)$, but there is a positive mass dropping out before $p_{i}\left(k, h, s_{0}\right)$. Consider a sequence of small positive numbers $\left(\epsilon_{n}\right)$ converging to 0 as $n \rightarrow \infty$ and sequences $\left(\pi_{n}^{1}\right),\left(\pi_{n}^{2}\right), \ldots,\left(\pi_{n}^{k-1}\right)$, where $\pi_{n}^{l}$ is the probability that player $i$ with value $s_{0}$ will end up in position $l$ if another player drops out at price $\left(p_{i}\left(k, h, s_{0}\right)-\epsilon_{n}\right)$. Let $B=\alpha_{1} s_{0}$, i.e., the maximum payoff that a player with value $s_{0}$ can possibly get in the auction. Now, if $\left(\pi_{n}^{k-1}\right)$ converges to 1 and $\left(\pi_{n}^{l}\right)$ converges to zero for all $l<k-1$, then, by an argument similar to the one at the end of Step 1(a), it is better for player $i$ of type $s_{0}$ to drop out at some time $p_{i}\left(k, h, s_{0}\right)-\epsilon .^{24}$ If $\left(\pi_{n}^{k-1}\right)$ does not converge to 1 , take the highest $l$ for which $\left(\pi_{n}^{l}\right)$ does not converge to zero, and take a subsequence of $\epsilon_{n}$ along which $\left(\pi_{n}^{l}\right)$ converges to some positive number $\rho$. Let $s_{1}$ be the value such that for a random draw of types of remaining players other than $i$, conditional on each draw being greater than $s_{0}$, the probability that at least one type is less than $s_{1}$ is equal to $\frac{\rho}{2}$ (i.e., $\prod_{j \neq i} \frac{1-F_{j}\left(s_{1}\right)}{1-F_{j}\left(s_{0}\right)}=1-\frac{\rho}{2}$ ). Clearly, $s_{1}>s_{0}$. Take a small $\epsilon_{n}$, and consider a subgame following some continuation of history $h, h_{l+2}$, where the $(l+2)^{n d}$ player drops out at $b_{l+2}, l+1$ players, including player $i$, remain, and player $i$ gets position $l$ with probability close to $\rho$ (and any position higher than $l$ with probability close to zero). Consider $p_{i}\left(l+1, h_{l+2}, s_{0}\right)$. There must exist a player, $j$, such that $p_{j}\left(l+1, h_{l+2}, s_{1}\right) \leq p_{i}\left(l+1, h_{l+2}, s_{0}\right)$. (Otherwise, the probability of player $i$ surviving until position $l$ is less than or close to $\frac{\rho}{2}$, and thus cannot be close to $\rho$.) But then, by an argument similar to the one in Step 1(a), in this subgame it is strictly better for player $i$ to drop out slightly earlier than $p_{i}\left(l+1, h_{l+2}, s_{0}\right)$ : Conditional on somebody else dropping out in between, the benefit is close to zero (the probability of getting a position better than $l$ times the highest possible benefit $B$ ), while the cost is close to a positive number (the payoff from being in position $l+1$ at price $b_{l+2}$ vs. the payoff from being in position $l$ at price at least $s_{1}-\frac{\alpha_{l+1}}{\alpha_{l}}\left(s_{1}-b_{l+2}\right)$-contradiction.

Step 2. In Step 1, we have shown that $p_{\max }(k, h, s) \equiv \max _{i} p_{i}(k, h, s)$ cannot be greater than $q(k, h, s)$, and therefore for any player $i$ and type $s \geq s_{\text {min }}, p_{i}(k, h, s) \leq q(k, h, s)$. Take some value $s>s_{\text {min }}$ and player $i$. Suppose $p_{i}(k, h, s)<q(k, h, s)$. Take some other player $j$. From Step 1 , we have $p_{j}\left(k, h, s_{\text {min }}\right) \leq q\left(k, h, s_{\text {min }}\right)<q(k, h, s)$, and therefore if player $i$ waited until $q(k, h, s)$ instead of dropping out at $p_{i}(k, h, s)$, the probability that someone dropped out in between would be positive, and hence the payoff would be strictly greater (by the definition of function $q($ ), player $i$ with value $s$ strictly prefers being in position $k-1$ or higher at any price less than $q(k, h, s)$ to being in position $k$ at price $\left.b_{k+1}\right)$, which is impossible in equilibrium. Hence, $p_{i}(k, h, s)=q(k, h, s)$ for all $s>s_{\min }$. By continuity, we also have $p_{i}\left(k, h, s_{\min }\right)=q\left(k, h, s_{\min }\right)$.

\footnotetext{
${ }^{24}$ If some other player drops out between $p_{i}\left(k, h, s_{0}\right)-\epsilon$ and $p_{i}\left(k, h, s_{0}\right)$, the benefit of staying longer tends to zero (it is at most $B\left(1-\pi^{k-1}\right)$ ), while the cost converges to a positive number (the difference between getting position $k$ at price $b_{k+1}$ and position $k-1$ at price $\left.p_{i}\left(k, h, s_{0}\right)\right)$.
} 

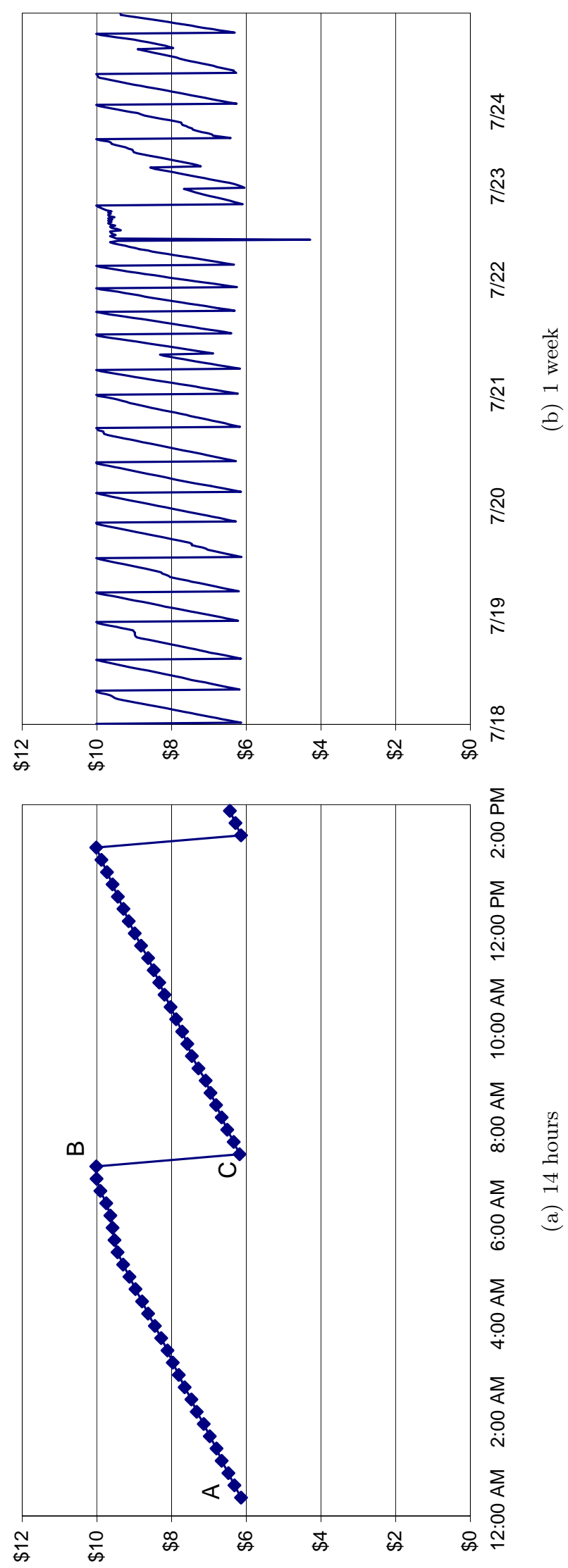

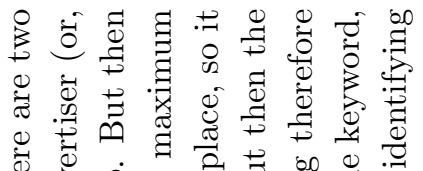

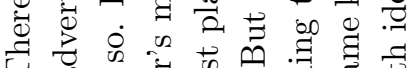

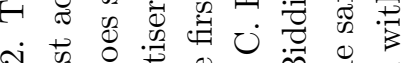

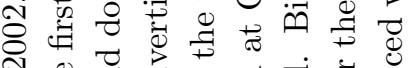

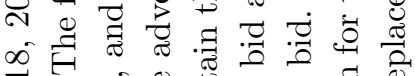

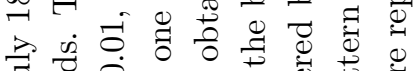

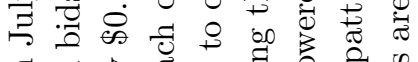

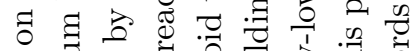

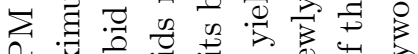

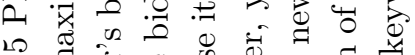

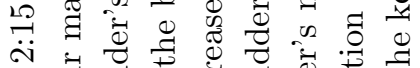

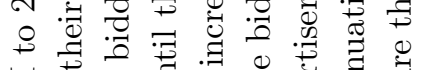

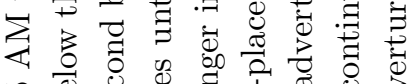

$\stackrel{ }{N}$

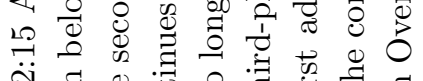

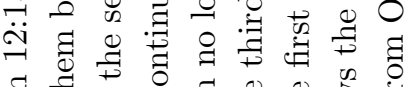
दี ॠ

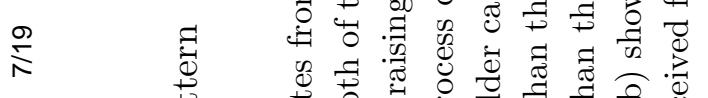

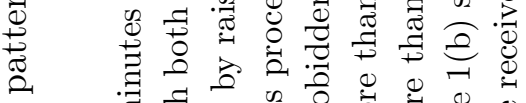

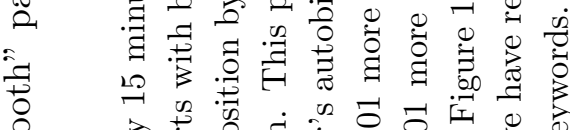

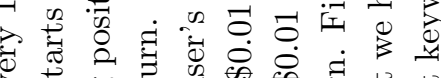

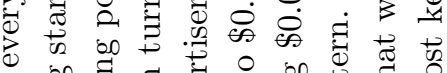

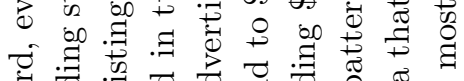

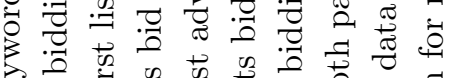

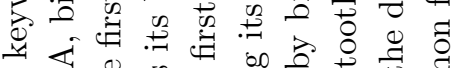

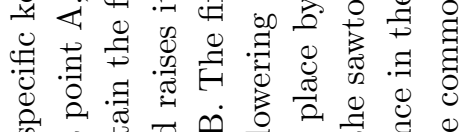
क त

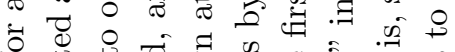

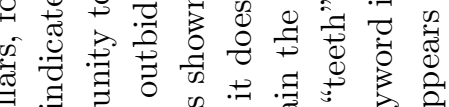

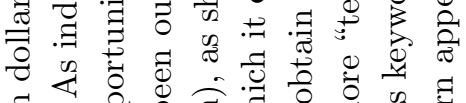

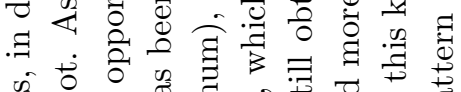
过

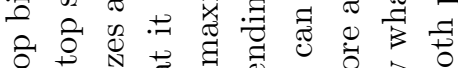
要

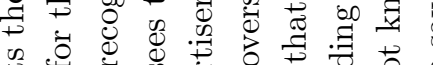
का

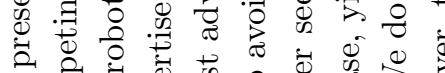
๙

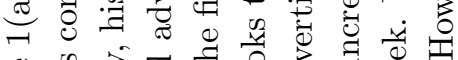
设

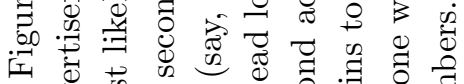

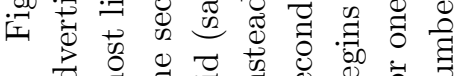

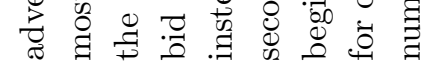

